\section{Schwache Blase, früher Tod?}

Senioren über 60, die nachts mehr als zweimal zur Toilette müssen, sind offenbar gesundheitlich gefährdet. In einer Studie war bei männlichen Nykturiepatienten die Mortalität gegenüber blasenstarken Alters- und Geschlechtsgenossen um $50 \%$ erhöht.

n einer aktuellen Studie wurden 2.447

Männer im Alter zwischen 40 und 79 Jahren über einen langen Zeitraum (im Mittel 17 Jahre) nachverfolgt und in Zweijahresabständen zur Häufigkeit nächtlicher Toilettengänge und im Verlauf der Studie neu aufgetretenen Gesundheitsproblemen befragt.

Im Gesamtkollektiv waren zunächst keine deutlichen Assoziationen bestimmter Erkrankungen mit dem Harndrang zu erkennen. Erwartungsgemäß zeichnete sich jedoch eine starke Altersabhängigkeit des Symptoms ab, sodass die Forscher verschiedene Altersgruppen bildeten. Bei den über 60-Jährigen war eine deutliche Assoziation der Nykturie mit einem frühzeitigen Tod zu beobachten, und zwar unabhängig von koronarer Herzkrankheit (KHK), Body-Mass-Index (BMI) und Einnahme urologischer Präparate: Die Sterberate innerhalb des Beobachtungszeitraums war um fast $50 \%$ erhöht. Dabei war sowohl das Hypertonieals auch das Diabetesrisiko unterm Strich bei den jüngeren Männern nur minimal größer, bei den älteren Männern sogar etwas niedriger als bei normaler Blasenfunktion. Insgesamt wurde bei $6,5 \%$ der Befragten nach Ablauf des Follow-up ein neu aufgetretener Diabetes mellitus diagnostiziert, ein neu aufgetretener Hyper- tonus bei $32 \%$. Die Nykturie ist zwar bekanntermaßen ein frühes Symptom einer Diabeteserkrankung, sie verschwindet jedoch in der Regel, wenn der Stoffwechsel gut eingestellt und für eine gute Diurese gesorgt ist.

Fazit: Mehr als zwei nächtliche Gänge zum WC können ein Prädiktor für eine koronare Herzkrankheit (KHK) oder einen früheren Tod sein. Frühere Studien hatten bereits einen Zusammenhang der Nykturie mit häufigeren Hüftfrakturen hergestellt. Dies führt die Autoren zu der Vermutung, dass die Senioren beim nächtlichen Austreten wohl leicht stürzen und sich dabei die Hüfte brechen, was in dieser Altersgruppe zur erhöhten Mortalität beitragen kann. Dr. Elke Oberhofer

Lightner DJ et al. Nocturia is associated with an increased risk of coronary heart disease and death. BJU Int 2012 doi: $10.1111 /$ j.1464-410X.2011.10806.x 